\title{
Study Of Some Real Definite Integrals Over A Unit Triangle Via Adaptive Scheme
}

\author{
Saumya Ranjan Jena ${ }^{1}$, Kumudini Meher ${ }^{2}$,Arjun Kumar Paul ${ }^{3}$ \\ ${ }^{1,2,3}$ Department of Mathematics, School of Applied Sciences \\ KIIT DT University, Bhubaneswar-751024, Odisha, India \\ Email:saumyafma@kiit.ac.in,kiran1920@gmail.com,apaulfma@kiit.ac.in
}

\begin{abstract}
In this note, an effective quadrature rule with an adaptive scheme has been implemented over the triangular domain. The double transformations have been used to transform the triangular surface into a standard square space. The new quadrature rule has been employed in adaptive scheme taking 5 numerical texts for getting the improved result over the constituent rule and an error analysis has been proposed.
\end{abstract}

Index Terms- Mixed quadrature rule, Degree of precision, Maclaurin's series, Error bound, Adaptive quadrature scheme.

\section{INTRODUCTION}

The applications of mixed quadrature rule for the approximation of real integrals $I(h)=\int_{u}^{v} h(l) d l$

have been used by several authors. The symmetric Gaussian quadrature formula for integrating arbitrary functions of two variables over triangular region was proposed by [1], [2, 3], [4]. Next to them the same symmetric quadrature rules in polar co-ordinates was given by [5]. The symmetric integration formula with higher order precision up to degree ten was given by [6]. The researchers [7], [8] considered the product formula which was derived from Gaussian quadrature in single variable. The researcher [9] has proposed an alternative integration formula for triangular domain on the basis of finite element method. Lastly, the mixed quadrature on real definite integrals with finite element methods has been suggested by [10].

In this paper we adopt an application of mixed quadrature with adaptive scheme over the specific triangular domain $l, m \in[0,1]$ and $\{l+m \leq 1\}$.Here we have implemented a transformation scheme to transform from $(l, m)$ space to $\{(p, q):-1 \leq p, q \leq 1\}$ through a standard square $\alpha, \beta \in[0,1]$ space. A new rule of precision-7 has been obtained by mixing two constituent rules each of precision 5 .Our said rule is superior to that of Clenshaw-Curtis 5-point rule and a good agreement has been reached to that of exact result in adaptive scheme.

For a real integrable function $\mathrm{h}$, an interval $[u, v]$ and a prescribed tolerance $\varepsilon$, it is desired to compute an approximation $D$ to the integral $I=\int_{u}^{v} h(l) d l$ so that $|D-I| \leq \varepsilon$.The basic principle for adaptive quadrature is the additive property of a definite integral of the form $D=E+F$ with the adaptive integration schemes $[11,12,13]$ where $D=$ $\int_{u}^{v} h(l) d l, E=\int_{u}^{w} h(l) d l, F=\int_{w}^{v} h(l) d l$ and $w$ is any point between $u$ and $v$ In adaptive integration, the points at which the integrand is evaluated are so chosen in such a way that depends on the nature of the integrand. The basic concept for an approximation of an integral $D$ is the sum of two integrals $E$ and $F$ within a specified tolerance $\varepsilon$.

\section{Adaptive algorithm}

The inputs to the algorithm are $0,1, n, h, r, \varepsilon$. Where $n$ is the number of intervals chosen and $\varepsilon$ is the stopping criterion.

Step-1: An approximation $I=\iint_{T} h(l, m) d l d m=\int_{0}^{1} d m \int_{0}^{1-m} h(l, m) d l=\iint_{R} h(\alpha, \beta) d \alpha d \beta$ $\alpha, \beta \in[0,1]$ is to be calculated.

Step-1: An approximation $I=\iint_{T} h(l, m) d l d m=\int_{0}^{1} d m \int_{0}^{1-m} h(l, m) d l=\iint_{R} h(\alpha, \beta) d \alpha d \beta$ $\alpha, \beta \in[0,1]$ is to be calculated.

Step-2: Taking $c=\frac{0+1}{2}$, the original square is divided into 4 sub-squares each of side $c=\frac{1}{2}$ unit.

Step-3: For each individual square if each $I_{i}$ satisfy $\left|I_{i}(h)-h(l, m)\right| \leq \varepsilon$, then $I=I_{1}+I_{2}+I_{3}+I_{4}$ If not then go to step-2 i.e. $c=\frac{1}{2^{2}}$ unit and if $I_{i}, i=1, \ldots . ., 4 \quad$ satisfy $\mid I_{i}(h)-h(l, m) \leq \varepsilon, \quad$ the process will be stopped and it is added with previous $I_{i}$. 


\section{E-ISSN: 2321-9637}

\section{Available online at www.ijrat.org}

Step-4: Continuing this process $r$-times for each subsquare of side $c=\frac{1}{2^{r}}$ and if $\left|I_{i}(h)-h(l, m)\right| \leq \varepsilon$ then stop. Otherwise repeat step-3 until to get $|I-h(l, m)| \leq \varepsilon$.

This paper is designed as follows. Sec-1 contains introduction, sec- 2 bears the construction of quadrature rule over the domain $\mathrm{T}$ in adaptive environment, sec-3 contains the construction of mixed rule taking the two constituent rules $R_{L 4}(h)$ and $R_{C C 5}(h)$. The error analysis and error bound are worked out in sec-4.The numerical verification of our proposed rule is experimented on some suitable real integrals and the comparison with the constituent $R_{C C 5}(h)$ rule in adaptive scheme sec-5.The conclusion follows in sec- 6 .

\section{GENERAL CONSTRUCTION OF} QUADRATURE OVER A TRIANGULAR REGION

$I(h)=\iint_{T} h(l, m) d l d m=\int_{0}^{1} d m \int_{0}^{1-m} h(l, m) d l$

Where $l=\alpha, \quad m=(1-\alpha) \beta$

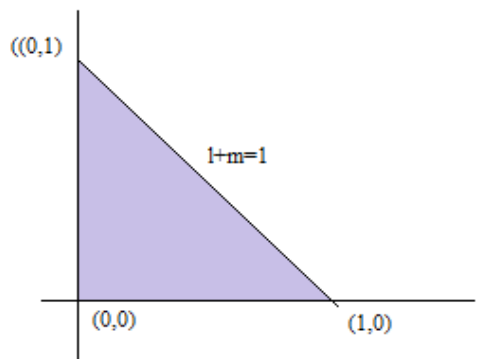

Fig-1 (Triangular region)

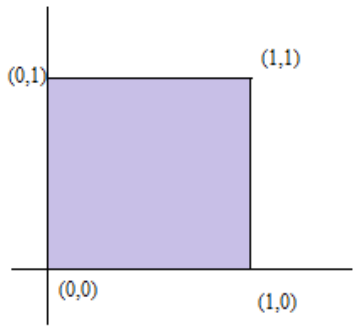

Fig-2 (Square region)

The Jacobian

$J=\frac{\partial(l, m)}{\partial(\alpha, \beta)}=1-\alpha$

Area $=d l d m=J d \alpha d \beta$
Now eqn (2.1) becomes,

$I(h)=\int_{0}^{1} \int_{0}^{1} h(\alpha,(1-\alpha) \beta)(1-\alpha) d \alpha d \beta$

Taking the substitution

$\alpha=\frac{1+p}{2}, \beta=\frac{1+q}{2}$

$d \alpha d \beta=\frac{\partial(\alpha, \beta)}{\partial(p, q)} d p d q=\frac{1}{4} d p d q$

Now using eqn (2.3) and eqn (2.4) in eqn (2.2),

$I(h)=\int_{-1}^{1} \int_{-1}^{1} h\left(\frac{1+p}{2}, \frac{(1-p)(1+q)}{4}\right)\left(\frac{1-p}{8}\right) d p d q$

Where $p, q \in[-1,1]$

\section{MIXED RULE}

In this section a new (mixed) rule has been established for approximation of

$I(h)=\int_{-1}^{1} \int_{-1}^{1} h(l, m) d l d m$

Clenshaw-Curtis five point rule 
International Journal of Research in Advent Technology, Vol.7, No.4, April 2019

E-ISSN: 2321-9637

Available online at www.ijrat.org

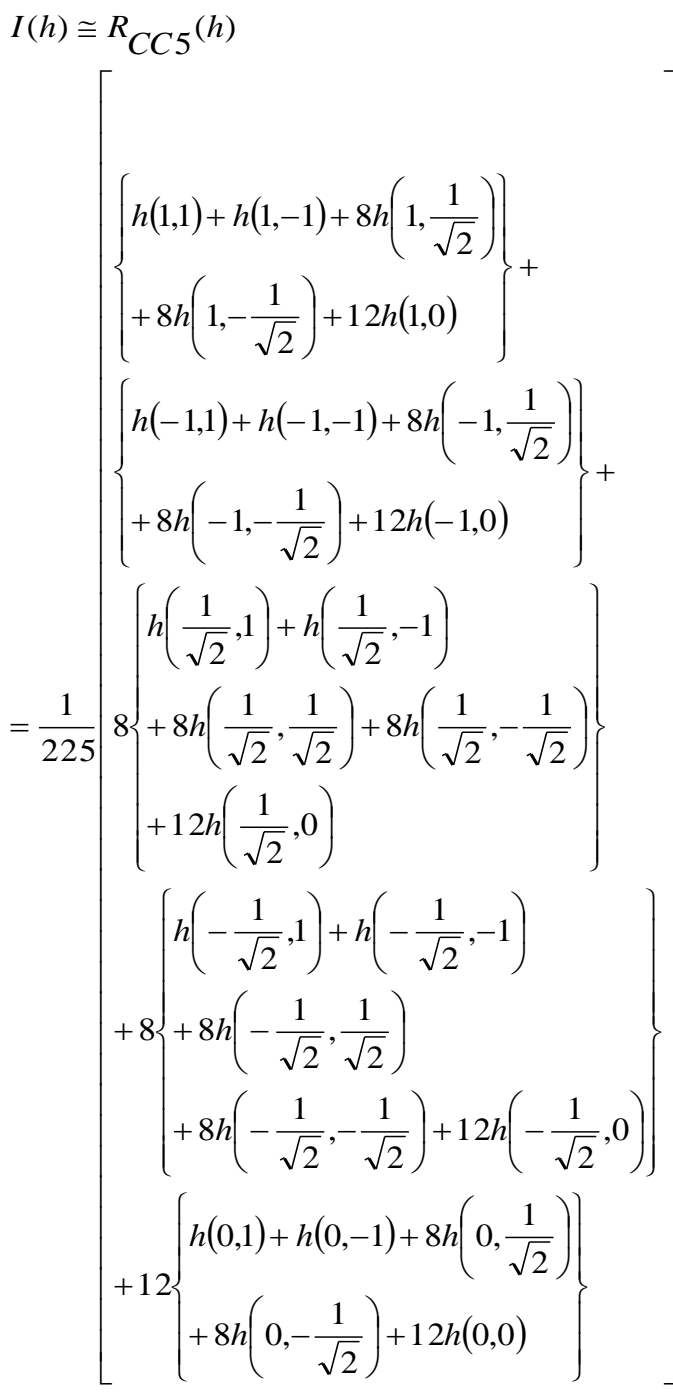

$$
\begin{aligned}
& \begin{array}{l}
\text { Lobatto } \\
I(h) \cong R_{L 4}(h)
\end{array} \\
& \left\{\begin{array}{l}
h(1,1)+5 h\left(1, \frac{1}{\sqrt{5}}\right)+ \\
5 h\left(1,-\frac{1}{\sqrt{5}}\right)+h(1,-1)
\end{array}\right\}+ \\
& \int_{5} h\left(\frac{1}{\sqrt{5}}, 1\right)+5 h\left(\frac{1}{\sqrt{5}}, \frac{1}{\sqrt{5}}\right) \\
& 5\left\{+5 h\left(\frac{1}{\sqrt{5}},-\frac{1}{\sqrt{5}}\right)+h\left(\frac{1}{\sqrt{5}},-1\right)\right\}^{+} \\
& =\frac{1}{36}\left\{\begin{array}{l}
5 h\left(-\frac{1}{\sqrt{5}}, 1\right)+5 h\left(-\frac{1}{\sqrt{5}}, \frac{1}{\sqrt{5}}\right) \\
+5 h\left(-\frac{1}{\sqrt{5}},-\frac{1}{\sqrt{5}}\right)+h\left(-\frac{1}{\sqrt{5}},-1\right)
\end{array}\right\}+ \\
& \left\{h(-1,1)+5 h\left(-1, \frac{1}{\sqrt{5}}\right)\right. \\
& +5 h\left(-1,-\frac{1}{\sqrt{5}}\right)+h(-1,-1)
\end{aligned}
$$

Expanding each term of eqn (3.2) and eqn (3.3) using Maclaurin's series,

$$
\begin{aligned}
& I(h)=R_{C C 5}(h)+E_{C C 5}(h) \\
& I(h)=R_{L 4}(h)+E_{L 4}(h)
\end{aligned}
$$

Where

$$
\begin{aligned}
& R_{C C 5}(h)=4 h_{0,0}(0,0)+\frac{2}{3}\left[h_{2,0}(0,0)+h_{0,2}(0,0)\right] \\
& +\frac{1}{30}\left[h_{4,0}(0,0)+h_{0,4}(0,0)\right] \\
& +\frac{1}{9} h_{2,2}(0,0)+\frac{1}{180}\left[h_{4,2}(0,0)+h_{2,4}(0,0)\right] \\
& +\frac{8}{15 \times 6 !}\left[h_{6,0}(0,0)+h_{0,6}(0,0)\right]+ \\
& \quad+\frac{1}{3600} h_{4,4}(0,0)+\frac{224}{45 \times 8 !}\left[\begin{array}{l}
h_{6,2}(0,0) \\
+h_{2,6}(0,0)
\end{array}\right] \\
& +\frac{2}{5 \times 8 !}\left[h_{8,0}(0,0)+h_{0,8}(0,0)\right]+\ldots
\end{aligned}
$$




$$
\begin{aligned}
& R_{L 4}(h)=4 h_{0,0}(0,0)+\frac{2}{3}\left[\begin{array}{l}
h_{2,0}(0,0) \\
+h_{0,2}(0,0)
\end{array}\right] \\
& +\frac{1}{30}\left[h_{4,0}(0,0)+h_{0,4}(0,0)\right] \\
& +\frac{1}{9} h_{2,2}(0,0)+\frac{1}{180}\left[h_{4,2}(0,0)+h_{2,4}(0,0)\right] \\
& +\frac{52}{75 \times 6 !}\left[h_{6,0}(0,0)+h_{0,6}(0,0)\right]+ \\
& +\frac{1}{3600} h_{4,4}(0,0)+\frac{1456}{225 \times 8 !}\left[\begin{array}{l}
h_{6,2}(0,0) \\
+h_{2,6}(0,0)
\end{array}\right] \\
& +\frac{84}{125 \times 8 !}\left[h_{8,0}(0,0)+h_{0,8}(0,0)\right]+\ldots
\end{aligned}
$$

We can write eqn (3.1) using Maclaurin's expansion

$$
\begin{aligned}
& I(h)=4 h_{0,0}(0,0)+\frac{2}{3}\left[\begin{array}{l}
h_{2,0}(0,0) \\
+h_{0,2}(0,0)
\end{array}\right] \\
& +\frac{1}{30}\left[h_{4,0}(0,0)+h_{0,4}(0,0)\right] \\
& +\frac{1}{9} h_{2,2}(0,0)+\frac{1}{180}\left[\begin{array}{l}
h_{4,2}(0,0) \\
+h_{2,4}(0,0)
\end{array}\right] \\
& +\frac{4}{7 !}\left[h_{6,0}(0,0)+h_{0,6}(0,0)\right]+ \\
& +\frac{1}{3600} h_{4,4}(0,0)+\frac{1}{7560}\left[\begin{array}{l}
h_{6,2}(0,0) \\
+h_{2,6}(0,0)
\end{array}\right] \\
& +\frac{4}{9 !}\left[h_{8,0}(0,0)+h_{0,8}(0,0)\right]+\ldots \\
& E_{C C 5}(h)=I(h)-R_{C C 5}(h) \\
& =\frac{4}{105 \times 6 !}\left[h_{6,0}(0,0)+h_{0,6}(0,0)\right] \\
& +\frac{16}{45 \times 8 !}\left[h_{6,2}(0,0)+h_{2,6}(0,0)\right] \\
& +\frac{2}{45 \times 8 !}\left[h_{8,0}(0,0)+h_{0,8}(0,0)\right] \\
& E_{L 4}(h)=I(h)-R_{L 4}(h) \\
& =-\frac{64}{75 \times 7 !}\left[h_{6,0}(0,0)+h_{0,6}(0,0)\right] \\
& \left.-\frac{256}{25 \times 9 !\left[h_{6,2}(0,0)\right.}+h_{2,6}(0,0)\right] \\
& -\frac{256}{125 \times 9 !}\left[h_{8,0}(0,0)+h_{0,8}(0,0)\right] \\
& +
\end{aligned}
$$

Now multiplying $\left(\frac{16}{5}\right)$ in eqn (3.4) and adding eqn (3.4) and eqn (3.5) we get,

$I(h)=R_{C C 5 L 4}(h)+E_{C C 5 L 4}(h)$

Where

$$
\begin{aligned}
& R_{C C 5 L 4}(h)=\frac{1}{21}\left[16 R_{C C 5}(h)+5 R_{L 4}(h)\right] \\
& E_{C C 5 L 4}(h)=\frac{1}{21}\left[16 E_{C C 5}(h)+5 E_{L 4}(h)\right]
\end{aligned}
$$

Now, $E_{C C 5 L 4}(h)$ can be obtained by substituting eqn (3.9) and eqn (3.10) in eqn (3.13),

\section{ERROR ESTIMATION}

Theorem-4.1 The error $E_{C C 5 L 4}(h)=I-R_{C C 5 L 4}(h)$

$$
\begin{gathered}
\text { and }\left|E_{C C 5 L 4}(h)\right| \leq \frac{128 K}{2205 \times 6 !} \text { where } \\
K=\max _{\substack{-1 \leq x \leq 1 \\
-1 \leq y \leq 1}}\left|h_{7,0}(l, *)+h_{0,7}(*, m)\right|
\end{gathered}
$$

Proof: We have

$$
E_{C C 5}(h)=\frac{4}{105 \times 6 !}\left[h_{6,0}\left(\eta_{2}, 0\right)+h_{0,6}\left(0, \eta_{2}\right)\right]
$$

$$
E_{L 4}(h)=-\frac{64}{525 \times 6 !}\left[h_{6,0}\left(\eta_{1}, 0\right)+h_{0,6}\left(0, \eta_{1}\right)\right]
$$

where $\eta_{1}, \eta_{2} \in[-1,1]$

$$
\begin{aligned}
& E_{C C 5 L 4}(h)=\frac{1}{21}\left[16 E_{C C 5}(h)+5 E_{L 4}(h)\right] \\
& =\frac{64}{2205 \times 6 !}\left[\begin{array}{l}
h_{6,0}\left(\eta_{2,}, 0\right)+h_{0,6}\left(0, \eta_{2}\right)-h_{6,0}\left(\eta_{1,}, 0\right) \\
-h_{0,6}\left(0, \eta_{1}\right)
\end{array}\right] \\
& =\frac{64}{2205 \times 6 !}\left[\int_{\eta_{1}}^{\eta_{2}} h_{7,0}(l, 0) d l+\int_{\eta_{1}}^{\eta_{2}} h_{0,7}(0, m) d m\right] \\
& =\frac{64}{2205 \times 6 !} \int_{\eta_{1}}^{\eta_{2}} \int_{\eta_{1}}^{\eta_{2}}\left[h_{7,0}(l, *)+h_{0,7}(*, m)\right] d l d m \\
& \left|E_{C C 5 L 4}(h)\right| \leq \frac{64 K}{2205 \times 6 !}\left|\eta_{2}-\eta_{1}\right| \\
& \text { for }\left|\eta_{2}-\eta_{1}\right| \leq 2(\text { C.Conte and D.Boor }[11])
\end{aligned}
$$

$$
E_{C C 5 L 4}(h)=-\frac{32}{1575 \times 8 !}\left[\begin{array}{l}
h_{8,0}(0,0) \\
+h_{0,8}(0,0)
\end{array}\right]
$$


Available online at www.ijrat.org

$\left|E_{C C 5 L 4}(h)\right| \leq \frac{128 K}{2205 \times 6 !}$

Where $K=\max _{\substack{-1 \leq x \leq 1 \\-1 \leq y \leq 1}}\left|h_{7,0}(l, *)+h_{0,7}(*, m)\right|$

$K$ gives the truncational error bound as $\eta_{1}, \eta_{2}$ are unknown points in $[-1,1]$

5. Numerical verification

Here we have taken 5 tests for our purpose. The integrals are

$$
\begin{aligned}
I_{1} & =\int_{0}^{1} \int_{0}^{1-l} \sin (l+m) d m d l \\
I_{2} & =\int_{0}^{1} \int_{0}^{1-l} e^{l+m} d m d l \\
I_{3} & =\int_{0}^{1} \int_{0}^{1-l} \cosh (l+m) d m d l \\
I_{4} & =\int_{0}^{1} \int_{0}^{1-l} \cos ^{2}(l+m) d m d l, \\
I_{5} & =\int_{0}^{1} \int_{0}^{1-l} e^{l} \cos m d m d l .
\end{aligned}
$$

$$
\text { Table-* }
$$

\begin{tabular}{|c|c|c|c|}
\hline $\begin{array}{l}\text { No of } \\
\text { Interva } \\
\text { ls } \\
\text { for } \\
R\end{array}$ & $\begin{array}{l}R_{C C 5 L 4}(h) \\
\text { by } \\
\text { adaptive } \\
\text { method }\end{array}$ & $\begin{array}{l}\text { No of } \\
\text { Intervals } \\
\text { for } \\
R_{C C 5 L 4}(h)\end{array}$ & $\begin{array}{l}\text { absolute error } \\
(\varepsilon)\end{array}$ \\
\hline 2 & 0.3011686 & 1 & $\varepsilon_{1}=0.000000002$ \\
\hline
\end{tabular}

(Numerical test for $R_{C C 5}(h)$ and $R_{C C 5 L 4}(h)$ with stopping criterion $\varepsilon$ )

\begin{tabular}{|l|l|}
\hline Exact value & $\begin{array}{l}R_{C C 5}(h) \\
\text { by adaptive method }\end{array}$ \\
\hline$I_{1}=0.301168678939757$ & 0.301168681730568 \\
\hline$I_{2}=1.000000000000000$ & 1.000000000027503 \\
\hline$I_{3}=0.632120558828558$ & 0.632120558803135 \\
\hline$I_{4}=0.300306002138028$ & 0.300305994129496 \\
\hline$I_{5}=0.668254268891505$ & 0.668254274236139 \\
\hline
\end{tabular}

Table-*

\begin{tabular}{|l|l|l|l|}
\hline & 78941508 & & \\
\hline 2 & $\begin{array}{l}0.9999999 \\
99997136\end{array}$ & 1 & $\varepsilon_{2}=0.00000000002$ \\
\hline 2 & $\begin{array}{l}0.6321205 \\
58828558\end{array}$ & 1 & $\varepsilon_{3}=0.00000000002$ \\
\hline 2 & $\begin{array}{l}0.3003060 \\
02056161\end{array}$ & 1 & $\varepsilon_{4}=0.000000008$ \\
\hline 3 & $\begin{array}{l}0.6682542 \\
68913063\end{array}$ & 1 & $\varepsilon_{5}=0.000000005$ \\
\hline
\end{tabular}

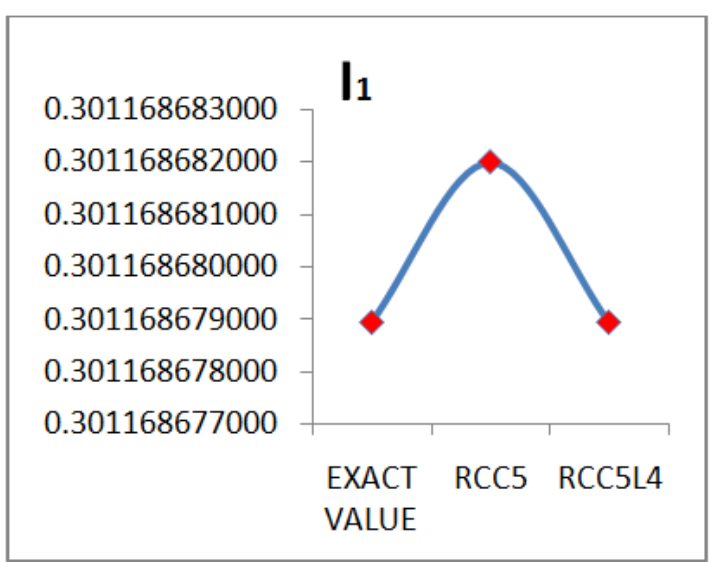

Comparison of Exact value with $R_{C C 5}(h)$ and $R_{C C 5 L 4}(h)$ of $I_{1}$.

\section{CONCLUSION}

The new (mixed) rule $R_{C C 5 L 4}(h)$ has made an agreement with that of exact value for different integrals as well as superior to $R_{C C 5}(h)$ rule from Table $-*$ and the graphs for $I_{1}$. The error and the error bound have been clearly reflected for our motto.

\section{REFERENCES}

[1]. P.C. Hammer, O.J. Marlowe and A.H. Stroud (1956), "Numerical integration over Simplexes and Cones", Math. Tables other Aids Computation, 10, 130-136.

[2] P.C. Hammer and A.H. Stroud (1956), "Numerical integration over Simplexes", Math. Tables other Aids Computation, 10,137-139.

[3]. P.C. Hammer and A.H. Stroud (1958), “ Numerical evaluation of multiple integrals" , Math. Tables other Aids Computation, 12, 272-280. 
[4] G.R. Cowper (1973),"Gaussian quadrature formulae for triangles", Int. J.Num.Math.Engg,7,405408.

[5]. J.N. Lyness and D. Jespersen (1975), "Moderate Degree Symmetric Quadrature Rules for the Triangle", J.Inst.Math.Applic, 15,19-32.

[6]. F.G. Lannoy (1977), "Triangular finite elements and numerical integration", Computer Struct, 7,613.

[7]. D.P. Laurie (1977), "Automatic numerical integration over a triangle", CSIR, spec. Rep. WISK 273, National institute for mathematical science, Pretoria.

[8]. M.E. Laursen and M. Gallert (1978), "Some criteria for numerically integrated matrices and quadrature formulae for triangles", Int.J.Num.Math.Engg, 12, 67-76.

[9]. C.T. Reddy and D.J. Shippy (1981), "Alternative integration formulae for triangular finite elements", Int.J.Num.Math.Engg, 17, 133-139.

[10]. S.R. Jena and R.B. Dash 2009) "Mixed quadrature of real definite integral over triangles", Pacific-Asian Journal of Mathematics, vol-3,1-2

[11]. S. Conte and C. De. Boor (1980), "Elementary Numerical Analysis”, Tata Mac-Graw Hill. 